\title{
Avaliação da posição condilar e disfunção temporomandibular em pacientes com má oclusão de Classe II submetidos à protrusão mandibular ortopédica
}

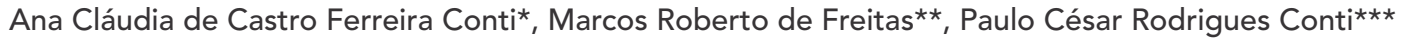

\begin{abstract}
Resumo
Objetivo: avaliar a participação da protrusão mandibular ortopédica e da posição condilar na prevalência de sinais e sintomas de disfunção temporomandibular (DTM). Metodologia: a amostra foi composta por 60 indivíduos divididos em 3 grupos, sendo o grupo I correspondente a indivíduos não tratados; o grupo II composto por jovens em tratamento com o Bionator; e o grupo III por jovens já tratados com este aparelho. Os indivíduos da amostra responderam a um questionário relativo aos principais sintomas de DTM, permitindo a classificação dos mesmos de acordo com a presença e severidade dessas disfunções. Esses jovens também se submeteram à avaliação da movimentação mandibular, palpação dos músculos mastigatórios e inspeção de ruídos articulares. Radiografias transcranianas padronizadas das ATMs direita e esquerda foram realizadas, para obtenção do grau de concentricidade condilar. Resultados: os testes ANOVA, Kruskal-Wallis e qui-quadrado foram utilizados para análise dos dados. De acordo com os resultados do questionário anamnésico, $66,67 \%$ da amostra foram classificados com ausência de DTM; 30\% com DTM leve e apenas 3,33\% com DTM moderada, sem diferença entre os grupos estudados ( $p>0,05$ ). Quanto à concentricidade condilar, o grupo II apresentou os valores de menor concentricidade (côndilos mais anteriorizados), com diferença estatisticamente significante em relação ao grupo I $(p<0,05)$. Uma associação entre a concentricidade condilar e a prevalência de DTM, no entanto, não foi encontrada. Conclusão: a protrusão ortopédica, apesar de alterar a posição dos côndilos, não aumentou a prevalência de DTM na população estudada.
\end{abstract}

Palavras-chave: Ortopedia. Disfunção temporomandibular. Posição condilar.

* Mestre e Doutora em Ortodontia pela FOB-USP.

** Professor Titular de Ortodontia do Departamento de Odontopediatria, Ortodontia e Saúde Coletiva da FOB-USP.

*** Professor Associado de Prótese do departamento de Prótese da FOB-USP. 


\section{INTRODUÇÃO}

As disfunções do sistema mastigatório têm alcançado um papel de destaque dentro do contexto odontológico das últimas décadas. A grande demanda de pacientes ${ }^{9}$ e a pequena quantidade de informação disponível na área fazem com que se multipliquem estudos na busca de respostas que possam contribuir com tratamentos mais eficazes, auxiliando também a esclarecer os fatores etiológicos envolvidos nessas disfunções.

A oclusão tem sido historicamente apontada como um fator etiológico das disfunções temporomandibulares (DTMs), assim como o tratamento ortodôntico. Por outro lado, também é sugerida a realização de tratamento ortodôntico para prevenção de sinais e sintomas de DTM, o que gera confusão entre os pesquisadores.

$\mathrm{Na}$ verdade, a realização de procedimentos de Ortodontia não parece predispor o indivíduo a apresentar $\mathrm{DTM}^{7,8}$.

Um dos procedimentos freqüentemente utilizados na fase de crescimento do indivíduo com má oclusão de Classe II é a estimulação do crescimento mandibular, utilizando-se de conceitos ortopédicos.

O avanço mandibular realizado com aparelhos do tipo Bionator ou Herbst causa uma anteriorização do côndilo dentro da fossa mandibular, obrigando todo o complexo côndilo/disco a acompanhar esse movimento durante certo período de tempo. Além disso, essa nova postura mandibular anteriorizada exige nova acomodação das fibras musculares. Parece claro, então, que esse tipo de terapia realmente leva a uma série de alterações no sistema estomatognático. No entanto, apesar dessas várias alterações, pouco ainda se conhece sobre o verdadeiro efeito da protrusão como agente predisponente, iniciador ou perpetuante das DTMs.

Com o intuito de contribuir para melhor entendimento dessas questões, este trabalho tem como finalidade avaliar a relação existente entre posição condilar, sinais e sintomas de DTM e protrusão mandibular ortopédica.

\section{REVISÃO DE LITERATURA}

Nas últimas décadas, muitas pesquisas foram realizadas para esclarecer a controvérsia sobre a participação do tratamento ortodôntico nas DTMs. Apontado como um fator etiológico das DTMs $^{29,44}$, o tratamento ortodôntico também é considerado benéfico, contribuindo para melhorar os sinais e sintomas de DTM ${ }^{18,19,20}$. Mas a grande maioria dos trabalhos $7,10,22,23,24,26$ concorda que o tratamento ortodôntico não predispõe os indivíduos a manifestarem sinais e sintomas de DTM, o que também não indica a realização de Ortodontia com o intuito de tratar pacientes acometidos por essas disfunções.

Porém, uma modalidade de tratamento comumente utilizada para correção das más oclusões de Classe II, empregando aparelhos ortopédicos que avançam a mandíbula, tem despertado o interesse dos pesquisadores, devido à alteração de todo o relacionamento côndilo-disco-fossa mandibular, decorrente dessa terapia.

Ao mesmo tempo que essa alteração da posição condilar pode ser danosa para as articulações temporomandibulares (ATMs), esses aparelhos são considerados como terapia das disfunções temporomandibulares, por alguns autores ${ }^{30}$.

Nesse contexto, o posicionamento condilar dentro da fossa mandibular, assim como os métodos de imagem da ATM utilizados para a sua avaliação, têm sido motivo de vários estudos. Alguns autores relataram a associação das relações não concêntricas côndilo-fossa à função anormal da ATM $^{34}$ assim como outros relacionaram a simetria bilateral do côndilo à ausência de sintomas clínicos em adultos ${ }^{28}$. Entretanto, o papel da posição condilar na etiologia das DTMs ainda permanece controvertido na literatura.

Mikhail e Rosen ${ }^{27}$, em 1979, determinaram a correlação entre sinais e sintomas de DTM e o posicionamento do côndilo na fossa mandibular. A amostra foi composta por três grupos: (1) pacientes com disfunção, (2) amostra aleatória e (3) pacientes que se submeteram a reabilitações 
oclusais. O último grupo foi subdividido em (a) pacientes que tinham sinais/sintomas antes da terapia e que tiveram melhora após o tratamento, (b) pacientes sem sinais antes e após tratamento, (c) pacientes que continuaram com os sinais após tratamento e (d) pacientes sem sinais antes da terapia e que apresentaram sintomatologia após tratamento. Os indivíduos foram submetidos a um exame clínico e radiográfico das ATMs, sendo que para obtenção das radiografias utilizou-se um dispositivo para fixar a cabeça. No grupo dos pacientes com disfunção, $88 \%$ tinham assimetria dos espaços articulares, um resultado muito similar aos $89,5 \%$ do grupo de inclusão aleatória, em que apenas $25 \%$ dos pacientes apresentavam sinais e sintomas de DTM. No terceiro grupo, nos pacientes sem melhoras após tratamento, 91\% tinham espaços articulares assimétricos, sendo $72,6 \%$ de posteriorização condilar. A concentricidade condilar não foi freqüente nos grupos estudados, independente dos pacientes apresentarem ou não sinais e sintomas de DTM.

Weinberg ${ }^{42}$, em 1979, avaliou o papel da posição condilar nas disfunções da ATM em 116 pacientes, dos quais 55 faziam parte do grupo com DTM e 61 do grupo controle. Dos que apresentavam disfunção, $71 \%$ tinham retrusão condilar ( $40 \%$ unilateral e $31 \%$ bilateral), $18,2 \%$ para anterior, $7,3 \%$ superior e $3,6 \%$ centrados. No grupo controle, $36 \%$ estavam retruídos $(21 \%$ unilateral e $14,7 \%$ bilateral), $10 \%$ estavam para superior, $23 \%$ centrados e $31 \%$ para anterior. Observou-se que a retrusão condilar foi vista com bastante freqüência no grupo controle, indicando que, quando se obtém a RC manipulando-se a mandíbula na posição mais posterior, não necessariamente se orienta o côndilo corretamente na fossa, como era comumente pensado.

Ainda em 1985, Pullinger et al..$^{34}$ efetuaram um estudo da posição condilar por meio de tomadas tomográficas das ATMs, em indivíduos assintomáticos. A amostra foi composta por 46 jovens, sendo 26 do gênero masculino e 20 do feminino, com média de idade de 24 anos. Esses indivíduos foram examinados e classificados como assintomáticos em relação a desordens mastigatórias, não apresentando história prévia de tratamento ortodôntico ou ajuste oclusal nem coroas nos molares. Tomografias lineares das ATMs direita e esquerda foram obtidas dos 46 jovens em posição de intercuspidação, e a concentricidade condilar foi mensurada de acordo com o método proposto ${ }^{33}$. A concentricidade condilar foi encontrada em aproximadamente 50 a $65 \%$ dos jovens, com uma grande variabilidade. A distribuição de côndilos não-concêntricos foi, significantemente, mais para anterior nos indivíduos do gênero masculino e mais para posterior nos do gênero feminino. Devido à detecção de não-concentricidade condilar em pessoas assintomáticas, concluiu-se que não se justifica qualquer forma de tratamento no intuito de se restabelecer a posição condilar.

Apesar da importância da posição condilar na ATM ainda não ter sido totalmente esclarecida, e a posição normal não ter sido definida, muitos estudos têm sido realizados no sentido de se guiar o côndilo a uma posição cêntrica na fossa mandibular, com o intuito de aliviar sintomas em pacientes com dor orofacial e desarranjo interno da ATM. Assim, Ren, Isberg e Westesson ${ }^{35}$, em 1995, verificaram a posição condilar em 34 articulações de pacientes assintomáticos e sem deslocamento de disco determinado por artrografia, e em 85 ATMs de pacientes com diferentes estágios de desarranjo interno. No referido estudo, os autores observaram grande variabilidade da posição condilar no grupo normal. Além disso, aproximadamente metade do número de articulações com deslocamento de disco com redução e dois terços das ATMs sem redução apresentaram côndilos posicionados posteriormente. Entretanto, os autores alertam que a posição condilar posterior não pode ser utilizada para diagnóstico de deslocamento de disco, devido ao grande número de côndilos posicionados anterior e centralmente 
em pacientes com desarranjo interno da ATM.

Em um estudo realizado por Boscolo, Almeida e Paganini ${ }^{4}$, também em 1998, o posicionamento condilar foi determinado com o auxílio de radiografias transcranianas corrigidas e tomografias lineares em 50 voluntários, entre 18 e 35 anos. Nesse estudo, os espaços articulares foram obtidos por meio de gabaritos. As médias dos espaços articulares encontradas pelos autores foram de 2,64mm para o espaço articular anterior e 2,26mm para o espaço posterior.

$\mathrm{O}$ estudo de Bonilla-Aragon et $\mathrm{al}^{3}{ }^{3}$, nesse mesmo ano, avaliou a posição do côndilo na fossa mandibular como predecessor do deslocamento do disco. Cinqüenta e dois pacientes assintomáticos e 130 sintomáticos foram avaliados por meio de tomografia linear e ressonância magnética bilateral da ATM, para se averiguar deslocamento de disco e posição condilar, utilizandose medições lineares. Os pacientes sintomáticos apresentavam dor articular havia, pelo menos, seis meses, com ou sem limitação de movimento mandibular ou ruído na articulação. Nesse estudo, os autores encontraram 36,5\% de côndilos anteriorizados, 40,4\% concêntricos e 23,1\% posteriorizados em pacientes assintomáticos. Os mesmos valores para pacientes sintomáticos com articulação normal observada na RM foram, respectivamente, 33,3\%, 41,4\%, 25,3\%. Pacientes com deslocamento de disco com redução apresentaram 23,1\% de côndilos anteriorizados, $35,6 \%$ concêntricos e $41,3 \%$ posteriorizados, enquanto pacientes com deslocamento de disco sem redução exibiram valores de 20,3\%, 31,3\% e $48,4 \%$, referentes às mesmas posições condilares. Com base nesses resultados, concluiu-se que a posição do côndilo não é um bom indicador da presença ou ausência de deslocamento de disco.

Nesse mesmo ano, Pereira ${ }^{32}$, objetivando comparar as estruturas da ATM em pacientes portadores e não-portadores de DTM, realizou ultra-sonografia estática e tomografia linear em uma amostra de 40 adolescentes, com idades en- tre 12 e 18 anos de idade. Esses jovens foram divididos em dois grupos: o grupo I composto por 20 jovens (10 de cada gênero) considerados livres de sinais e sintomas de DTM, com base no indice craniomandibular; o grupo II composto pelo mesmo número de participantes, também divididos igualmente em relação ao gênero, porém apresentando DTM de acordo com o índice empregado. $\mathrm{O}$ autor concluiu que o posicionamento condilar mostrou-se mais posteriorizado no grupo com DTM de maneira significativa quando comparado ao grupo I, sendo essa posição posterior mais freqüente nas jovens. Apesar desses resultados, o autor ressalta que com esse estudo não se deve inferir uma relação causaefeito entre a posteriorização condilar e a presença de DTM.

Parece clara a importância que se atribui ao posicionamento côndilo-disco-fossa para as DTMs, portanto, ao se empregar aparelhos ortopédicos para estimular o crescimento mandibular, altera-se esse relacionamento por um período de tempo. A permanência ou não da alteração e os possíveis prejuízos para esses pacientes, em termos de manifestação de sinais e sintomas de DTM, precisam ser investigados.

Com o intuito de verificar os riscos para as ATMs da terapia de avanço mandibular empregando aparelho fixo, Ruf e Pancherz ${ }^{36}$, em 2000, acompanharam 62 pacientes com má oclusão de Classe II, tratados com essa terapia. A avaliação constou de exame clínico e obtenção de imagens de ressonância magnética das ATMs. Durante o tratamento, que durou em média sete meses, foi detectada uma capsulite temporária da porção inferior da região retrodiscal, que não se manteve em todo o período de avaliação, até um ano pós-tratamento. Não foram detectadas sintomatologia muscular e alterações no posicionamento dos discos em pacientes que os apresentavam fisiologicamente localizados antes do tratamento. Esta terapia contribuiu no posicionamento dos discos nos casos em que estes se apresentavam 
parcialmente deslocados ao início, porém o tratamento não resultou em melhora nos casos de deslocamento total do disco. Os autores ressaltaram que, em curto prazo, a terapia com o Herbst não provoca efeitos deletérios nas articulações.

Para desvendar os efeitos nas articulações com a utilização de um aparelho ortopédico removível (o ativador), Ruf, Wüsten e Pancherz ${ }^{37}$ em 2002, avaliaram 30 pacientes com má oclusão de Classe II, tratados com este aparelho, com média de idade inicial de 11,4 anos. Utilizaram, para isso, o recurso de imagens por ressonância magnética da ATM, realizada com a boca fechada antes e após um ano de tratamento, com o ativador e exame clínico para verificar a presença de dor na articulação. Os autores relataram que o tratamento com o ativador não alterou um pré-posicionamento fisiológico côndilofossa. Entretanto essa terapia não contribuiu na recaptura dos discos deslocados para anterior. A presença de capsulite subclínica na região posterior do disco foi um achado relevante nesse estudo.

A escassez de trabalhos com amostras de pacientes que se submeteram à protrusão mandibular ortopédica, e foram avaliados em relação à prevalência de DTM e ao posicionamento condilar, mantém a dúvida sobre os riscos dessa terapia no desencadeamento de sinais e sintomas de DTM. Com o objetivo de esclarecer esta questão, este trabalho se destina a avaliar as relações do posicionamento condilar e das DTMs com a realização de avanço mandibular ortopédico.

\section{MATERIAL E MÉTODOS}

A amostra foi composta por 60 indivíduos divididos em 3 grupos iguais, sendo o grupo I correspondente a indivíduos com má oclusão de Classe II não-tratados; o grupo II composto por jovens em tratamento com o bionator; e o grupo III por jovens já tratados com este aparelho.

Solicitou-se aos pacientes o preenchimento de fichas contendo informações pessoais e questões sobre sintomas relativos às DTMs. Este questionário, composto por 10 questões referente aos sintomas de DTM, foi desenvolvido baseado em fichas pré-existentes ${ }^{7,8,11,15,16}$ e aplicado aos pacientes, permitindo a classificação dos indivíduos em relação à presença e severidade de disfunção.

\section{Questionário anamnésico}

1. Você sente dificuldade em abrir a boca?

2. Você sente dificuldade em movimentar sua mandíbula para os lados?

3. Você sente desconforto ou dor muscular quando mastiga?

4. Você sente dores de cabeça, com freqüência?

5. Você sente dores no pescoço e/ou ombros?

6. Você sente dores de ouvido ou próximo a ele?

7. Você percebe algum ruído na ATM?

8. Você considera sua mordida "normal"?

9. Você usa apenas um lado de sua boca para mastigar?

10. Você sente dores na face, ao acordar?

Para as questões do questionário anamnésico foi oferecida a possibilidade de três respostas: "sim", "não" ou "às vezes". Para cada resposta foi atribuído um valor. A somatória dos valores obtidos permitiu a classificação da amostra em relação à DTM (Índice de DTM). Valores de 0 a 3 indicam ausência de DTM; valores de 4 a 8 a presença de DTM leve; 9 a 14 DTM moderada; e DTM severa quando a somatória dos valores das respostas se encontrava entre 15 e 23.

A avaliação das ATMs direita e esquerda foi realizada, sendo os movimentos mandibulares os primeiros itens analisados. Observou-se o grau de abertura máxima ativa (incluindo o trespasse), lateralidade direita e esquerda e a protrusão.

A presença de ruídos articulares a partir da 
inspeção das ATMs direita e esquerda foi também avaliada. Essa avaliação foi efetuada com palpação digital, enquanto o paciente realizava movimentos de abertura e fechamento mandibular. A presença de sintomatologia dolorosa na ATM também foi verificada com palpação das articulações, porém empregando pressão de 900 gramas ${ }^{2}$.

Para o exame da palpação muscular, os seguintes músculos foram considerados: temporal anterior, médio e posterior; origem, inserção e corpo do masseter superficial; masseter profundo; digástrico posterior; esternocleidomastóideo e trapézio superior. A pressão da palpação foi de aproximadamente 1.500 gramas $^{8}$. A presença de dor era constatada diante do reflexo palpebral e/ou questionamento ao paciente.

Uma avaliação dentária e oclusal foi executada, considerando a presença de desvios das posições de MIH e RC, presença e tipos de guia lateral e guia anterior, presença de mordida cruzada posterior e mordida aberta, assim como a severidade da má oclusão de Classe II.

O cálculo da concentricidade condilar realizado pela equação de Pullinger e Hollender ${ }^{33}$, empregando as mensurações dos espaços articulares anterior e posterior foi realizado. Os espaços articulares foram obtidos de mensurações nas radiografias transcranianas padronizadas, sendo que para o espaço articular anterior (A) foi considerado o menor espaço articular entre o côndilo e a parede anterior da fossa mandibular e para o espaço articular posterior (P) foi considerado o menor espaço articular entre o côndilo e a parede posterior da fossa mandibular (Fig. 1).

Valores negativos indicam um posicionamento condilar posterior, enquanto valores percentuais positivos indicam côndilos anteriorizados em relação à fossa mandibular. Valor "zero" indica a concentricidade absoluta do côndilo.

ANOVA a um critério, teste de Kruskal-Wallis e qui-quadrado foram utilizados para análise dos dados.

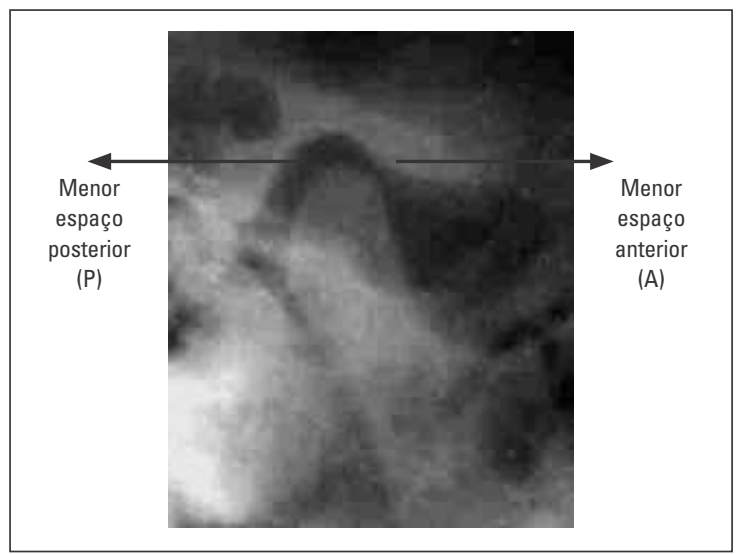

FIGURA 1 - Imagem transcraniana digitalizada.

\section{Cálculo da concentricidade condilar}

$$
\frac{\mathrm{P}-\mathrm{A}}{\mathrm{P}+\mathrm{A}} \times 100 \%
$$

\section{RESULTADOS}

$\mathrm{Na}$ amostra total, composta por 60 indivíduos, a média de idade foi de 12,58 $\pm 3,72$ anos, sendo a idade mínima de 7 e a máxima de 23 anos.

Verificou-se a prevalência de DTM nos três grupos estudados, de acordo com o questionário anamnésico descrito anteriormente (Tab. 1, Fig. 2). De acordo com o teste de Kruskal-Wallis, não houve diferença entre a presença de um dos subtipos de DTM nos diferentes grupos estudados.

A tabela 2 mostra os valores absolutos e percentuais do número de indivíduos que apresentaram dor à palpação na ATM, em pelo menos um ponto (dentre os aspectos lateral e posterior das ATMs esquerda e direita).

A tabela 3 apresenta os valores absolutos e percentuais do número de indivíduos que apresentaram, pelo menos, 3 pontos de dor à palpação dos músculos mastigatórios (porções anterior, média e posterior do músculo temporal, porções superficial e profunda do músculo masseter e digástrico posterior).

Uma não associação estatisticamente significante também foi encontrada quando avaliada a relação entre a discrepância existente entre as posições 
Tabela 1 - Prevalência de DTM nos diferentes grupos (valores absolutos e percentuais).

\begin{tabular}{lllll}
\hline DTM & grupo I & grupo II & grupo III & total \\
\hline ausente & $10(50 \%)$ & $15(75 \%)$ & $15(75 \%)$ & $40(66,67 \%)$ \\
leve & $10(50 \%)$ & $5(25 \%)$ & $3(15 \%)$ & $18(30 \%)$ \\
moderada & $0(0 \%)$ & $0(0 \%)$ & $2(10 \%)$ & $2(3,33 \%)$ \\
total & $20(100 \%)$ & $20(100 \%)$ & $20(100 \%)$ & $60(100 \%)$ \\
\hline
\end{tabular}

Tabela 2 - Valores absolutos e percentuais do número de indivíduos com e sem dor à palpação da ATM.

\begin{tabular}{lllll}
\hline & grupo I & grupo II & gupo III & total \\
\hline $\begin{array}{l}\text { ausência } \\
\text { de dor }\end{array}$ & $16(80 \%)$ & $13(65 \%)$ & $13(65 \%)$ & $42(70 \%)$ \\
$\begin{array}{l}\text { presença } \\
\text { de dor }\end{array}$ & $4(20 \%)$ & $7(35 \%)$ & $7(35 \%)$ & $18(30 \%)$ \\
total & $20(100 \%)$ & $20(100 \%)$ & $20(100 \%)$ & $60(100 \%)$ \\
\hline
\end{tabular}

de RC e MIH e a presença ou severidade de DTM (Tab. 4).

Os valores médios das mensurações dos espaços articulares para as ATMs direita e esquerda foram divididos de acordo com os grupos I, II e III (Tab. 5).

Para efeito da análise estatística e comparação entre os grupos estudados, foi considerado o lado de menor concentricidade, avaliado de acordo com os critérios já detalhados anteriormente. Os valores são expressos em percentuais, sendo $-100 \%$ o valor de posicionamento posterior absoluto, "zero" de concentricidade absoluta e $100 \%$ de posicionamento anterior máximo.

De acordo com o teste ANOVA a um critério, houve diferença estatisticamente significativa entre os grupos estudados (I - pré-tratamento, II - em tratamento e III - pós-tratamento) quanto à concentricidade condilar $(\mathrm{p}=0,032)$. Após a realização do teste de Tukey para comparações múltiplas, verificou-se que houve diferença entre os grupos I (prétratamento) e II (em tratamento) (Tab. 6).

Não houve correlação entre a soma total dos valores do questionário anamnésico que compuseram

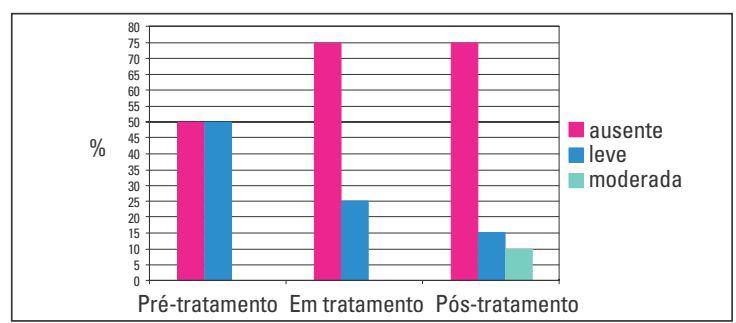

FIGURA 2 - Prevalência de DTM nos diferentes grupos (valores absolutos e percentuais).

Tabela 3 - Valores absolutos e percentuais do número de indivíduos com e sem dor à palpação dos músculos mastigatórios.

\begin{tabular}{lllll}
\hline & grupo I & grupo II & grupo III & total \\
\hline $\begin{array}{l}\text { ausência } \\
\text { de dor }\end{array}$ & $19(95 \%)$ & $19(95 \%)$ & $16(80 \%)$ & $54(90 \%)$ \\
$\begin{array}{l}\text { presença } \\
\text { de dor }\end{array}$ & $1(5 \%)$ & $1(5 \%)$ & $4(20 \%)$ & $6(10 \%)$ \\
\begin{tabular}{l} 
total \\
\hline
\end{tabular} & $20(100 \%)$ & $20(100 \%)$ & $20(100 \%)$ & $60(100 \%)$ \\
\hline
\end{tabular}

o índice de DTM e a concentricidade condilar para toda a amostra (Coeficiente de Spearman $=-0,197$ ) (Tab. 3).

\section{DISCUSSÃO}

Os aspectos oclusais foram considerados os principais fatores etiológicos das Disfunções Temporomandibulares, por muito tempo. Apesar do grande número de publicações atuais ${ }^{38,39}$ pertinentes a esta área, considerando limitada a participação dos mesmos na etiologia das DTMs, a controvérsia ainda permanece em relação à real participação desses fatores como agentes causadores de problemas articulares e musculares.

Os tratamentos ortodôntico e ortopédico, por influenciarem as relações oclusais ou, melhor, por alterarem o relacionamento maxilomandibular, também foram apontados como possiveis desencadeadores de DTM.

No caso dos aparelhos ortopédicos para correção das más oclusões de Classe II, a construção dos mesmos em uma posição anteriorizada da mandíbula ocasionaria uma alteração da posição condilar e da relação côndilo-disco. A manutenção da posi- 
Tabela 4 - Valores absolutos e percentuais do número de indivíduos com diferentes posicionamentos condilares sagitais nos diferentes grupos de DTM.

\begin{tabular}{lllll}
\hline & $\begin{array}{l}\text { ausência } \\
\text { de DTM }\end{array}$ & $\begin{array}{l}\text { DTM } \\
\text { leve }\end{array}$ & $\begin{array}{l}\text { DTM } \\
\text { moderada }\end{array}$ & Total \\
\hline $\begin{array}{l}\text { Disc RC-MIH } \\
\text { de 0 a 2mm }\end{array}$ & $37(92,5 \%)$ & $17(94,44 \%)$ & $2(100 \%)$ & $56(93,33 \%)$ \\
$\begin{array}{l}\text { Disc RC-MIH } \\
\text { de 2 a 4mm }\end{array}$ & $3(7,5 \%)$ & $1(5,56 \%)$ & $0(0 \%)$ & $4(6,67 \%)$ \\
TOTAL & $40(100 \%)$ & $18(100 \%)$ & $2(100 \%)$ & $60(100 \%)$ \\
\hline
\end{tabular}

Tabela 6 - Médias percentuais seguidas dos desvios-padrão dos valores de concentricidade condilar nos grupos estudados.

\begin{tabular}{ccc}
\hline & média $(\%) \pm$ desvio-padrão & p \\
\hline grupo I - pré-tratamento & $0,050 \pm 18,752$ & \\
grupo II - em tratamento & $17,450 \pm 23,449$ & $0,032^{*}$ \\
grupo III - pós-tratamento & $13,550 \pm 21,693$ & \\
\hline
\end{tabular}

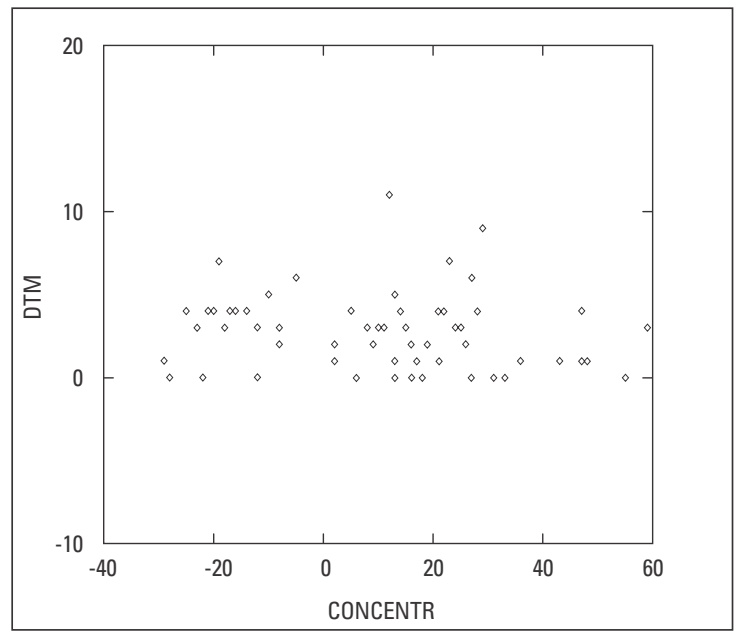

FIGURA 3 - Correlação entre a concentricidade condilar e o índice de DTM.

ção condilar concêntrica nas fossas mandibulares é defendida por alguns autores ${ }^{40,41}$, que a consideram importante para a normalidade da ATM. Em contrapartida, Pullinger et al. ${ }^{34}$ alegam que a ausência de concentricidade condilar não está associada ao aparecimento de sinais e sintomas de DTM, devido à grande variabilidade nesse posicionamento encontrada em indivíduos assintomáticos.

Para obtenção de respostas claras sobre o efei-
Tabela 5 - Valores (em mm) das médias e desvios-padrão acompanhados dos valores máximos e mínimos dos espaços articulares anterior (A) e posterior (P), para os lados direito e esquerdo dos grupos I, II e III.

\begin{tabular}{ccccccc}
\hline & $\begin{array}{c}\text { (A) } \\
\text { grupo } \\
\end{array}$ & $\begin{array}{c}\text { I } \\
\text { grupo } \\
\text { I }\end{array}$ & $\begin{array}{c}\text { (A) } \\
\text { grupo } \\
\text { II }\end{array}$ & $\begin{array}{c}\text { (P) } \\
\text { grupo } \\
\text { II }\end{array}$ & $\begin{array}{c}\text { (A) } \\
\text { grupo } \\
\text { III }\end{array}$ & $\begin{array}{c}\text { (P) } \\
\text { grupo } \\
\text { III }\end{array}$ \\
\hline média & 1,83 & 1,94 & 1,82 & 2,42 & 1,77 & 2,19 \\
d.p. & 0,48 & 0,36 & 0,41 & 0,65 & 0,40 & 0,66 \\
mínimo & 1,1 & 1,0 & 1,0 & 1,4 & 1,0 & 1,5 \\
máximo & 3,3 & 2,6 & 2,6 & 3,9 & 2,5 & 4,4 \\
\hline
\end{tabular}

to desses aparelhos ortopédicos na manifestação de sinais e sintomas de DTM, esse estudo propôs-se a avaliar a posição condilar em indivíduos submetidos à protrusão mandibular ortopédica e qual a relação com as diferentes manifestações de DTM.

Por vários anos, a discrepância das posições de RC e MIH sustentou a teoria oclusal de etiologia das DTMs. Variações entre essas posições de até $2 \mathrm{~mm}$ foram constatadas em 93,3\% da amostra, enquanto $6,67 \%$ dos indivíduos exibiram diferenças entre essas posições de 2 a $4 \mathrm{~mm}$. Levando-se em consideração que apenas discrepâncias maiores que $4 \mathrm{~mm}$ dobrariam as chances de se desenvolver $\mathrm{DTM}^{26}$, e que neste estudo não se detectaram variações dessa ordem, os resultados obtidos mostrando uma não associação entre essa variável e a prevalência de DTM podem ser justificados.

De acordo com os resultados obtidos com o questionário anamnésico, $66,67 \%$ da amostra apresentaram-se com ausência de DTM, 30\% com DTM leve, enquanto apenas 3,33\% foram classificados com DTM moderada. Com a utilização deste questionário, nenhum indivíduo da amostra foi considerado como apresentando DTM severa. Tais valores foram obtidos de acordo com o índice proposto e modificado por diversos autores ${ }^{8,11,16,17}$, com o objetivo de facilitar a classificação de uma amostra em relação à presença e severidade de DTM.

Os valores de prevalência de DTM deste estudo foram próximos aos encontrados por Conti et al. 7 : 62,5\% com ausência de DTM, 34\% com DTM leve 
e 3,5\% com DTM moderada.

Quando se comparou a presença e severidade de DTM entre os três grupos, não se encontrou uma diferença estatisticamente significante entre eles. Se a prevalência de DTM nos três grupos foi similar, o fato dos indivíduos estarem em tratamento com terapia de avanço mandibular, ou mesmo de já terem finalizado esse tratamento, não aumenta as chances dos mesmos apresentarem sintomas de DTM.

Com a terapia de avanço mandibular e deslocamento do côndilo das fossas mandibulares, aventouse a hipótese de desencadeamento de dor e ruídos articulares. Quando os valores obtidos durante o exame de palpação das ATMs foram comparados entre os grupos I, II e III, não foram encontradas diferenças entre eles, ou seja, a sensibilidade à palpação das ATMs foi similar, independente dos indivíduos se encontrarem com os côndilos terapeuticamente mais anteriorizados ou não. A freqüência de pelo menos um ponto sensível nos aspectos lateral e posterior das ATMs bilateralmente foi de $20 \%$ para o grupo I, $35 \%$ para o II e $35 \%$ para o grupo III.

Já a sensibilidade à palpação muscular, considerada como o sinal mais comum em estudos epidemiológicos, pode ser usada como recurso diagnóstico na detecção de DTM ${ }^{40}$.

O fator também supostamente relacionado à protrusão mandibular seria a presença de dores na musculatura mastigatória, devido a um posicionamento anormal da mandíbula.

Quando se considerou a freqüência de pontos sensiveis na musculatura mastigatória entre os grupos I, II e III, os resultados mostraram uma similaridade destes valores entre os grupos.

Henrikson, Nilner e Kurol ${ }^{19}$, em estudo longitudinal onde pacientes tratados ortodonticamente de má oclusão de Classe II foram comparados a uma amostra com a mesma má oclusão não tratada e um grupo controle de oclusão normal, relataram uma melhora na sensibilidade muscular.

Já Pancherz ${ }^{31}$, avaliando os efeitos do aparelho Herbst em pacientes Classe II, relatou que o número de pacientes com sensibilidade à palpação muscular dobrou nos três primeiros meses de uso do aparelho, apesar desses sintomas praticamente desaparecerem ao final do tratamento.

Outro parâmetro avaliado na presente pesquisa foi a concentricidade condilar. $\mathrm{O}$ posicionamento condilar tem sido, historicamente, considerado um dos critérios para se diagnosticar e tratar pacientes com sinais e sintomas de DTM.

A relação entre a concentricidade condilar e os desarranjos internos da ATM tem sido discutida, sendo que alguns autores ${ }^{41,42}$ creditam à ausência de concentricidade condilar, causada pelo mau posicionamento dos côndilos, um papel importante no desencadeamento de desordens intra-articulares.

Nesse contexto, a realização de procedimentos ortodônticos que possam interferir no relacionamento côndilo-fossa-disco torna-se potencialmente preocupante. Wyatt ${ }^{44}$ apontou a utilização de elásticos intermaxilares, mentoneiras e a retração dos incisivos superiores ${ }^{8}$ como procedimentos de risco para desencadear processo ${ }^{8}$ intra-articulares, devido à posteriorização dos côndilos ${ }^{6}$ Em contrapartida, trabalhos que avaliaram a posição condilar em pacientes tratados ortodonticamente não confirmam a hipótese acima ${ }^{12,13,23,24,25}$. Para aumentar a controvérsia, Carlton e $\mathrm{Nanda}^{5}$, quando avaliaram pacientes tratados ortodonticamente, relataram uma melhora na concentricidade condilar após o término da terapia.

Considerando toda a amostra, as médias do espaço articular anterior foram: do lado direito $1,7 \mathrm{~mm}$ e lado esquerdo $1,8 \mathrm{~mm}$, sendo os respectivos valores para o espaço articular posterior direito de $2,3 \mathrm{~mm}$ e $2,1 \mathrm{~mm}$ para o esquerdo. Estes valores foram similares aos de Ismail e Rokni ${ }^{21}$, ao compararem os espaços articulares mensurados em posições de RC e MIH, em pacientes Classe I, assintomáticos. Estes autores obtiveram para os espaços articulares anterior 2,1 $\mathrm{mm}$ bilateralmente, para o posterior lado direito $2,1 \mathrm{~mm}$ e esquerdo $2,3 \mathrm{~mm}$.

Os valores dos espaços articulares também foram considerados nos três grupos, isoladamente. Esses valores médios para o grupo I foram de $1,8 \mathrm{~mm}$ 
e $1,9 \mathrm{~mm}$ para os espaços anterior $(\mathrm{A})$ e posterior (P), respectivamente; para o grupo II $1,8 \mathrm{~mm}$ para A e $2,4 \mathrm{~mm}$ para $\mathrm{P}$, sendo esses valores de $1,8 \mathrm{~mm}$ e 2,2mm para A e P no grupo III. Na análise destes dados, observa-se um aumento do espaço posterior nos grupos II e III, que são os grupos em tratamento e tratados com avanço mandibular, indicando um posicionamento mais anteriorizado do côndilo, mesmo com os valores semelhantes do espaço anterior. Talvez a similaridade nas médias dos espaços anteriores entre os grupos ocorra em conseqüência de uma reabsorção da parede posterior da eminência articular em decorrência ao deslocamento anterior da mandíbula.

A posição condilar pode ser inferida baseada nos valores desses espaços articulares que separam os limites do côndilo e da fossa mandibular. Todavia, para facilitar a determinação da concentricidade condilar uma fórmula proposta por Pullinger e Hollender ${ }^{33}$ foi empregada.

Quando as médias percentuais da concentridade condilar entre os grupos foi comparada, constatouse uma diferença estatisticamente significativa do grupo II para o grupo I, ou seja, os indivíduos do grupo II apresentavam os valores de menor concentricidade (côndilos mais anteriorizados) em relação aos indivíduos do grupo I.

Um posicionamento mais anteriorizado dos côndilos também foi citado por Ruf e Pancherz ${ }^{36}$ em amostras de pacientes Classe II tratados com o aparelho de Herbst, e em outro estudo ${ }^{31}$ com pacientes tratados com o ativador. Nos dois estudos, o método de avaliação da posição condilar foi por meio de imagens de ressonância magnética. Essa tendência de anteriorização condilar foi detectada na remoção do aparelho, mas não se manteve quando os pacientes foram avaliados um ano após a finalização do tratamento. Já a posição condilar, analisada antes e após o tratamento, no estudo com ativador, manteve-se dentro dos níveis fisiológicos, apesar de uma tendência à anteriorização condilar prévia ao tratamento. Essa tendência também foi observada no presente estudo. Ou seja, os pacientes
Classe II já apresentam, naturalmente, os côndilos anteriorizados. Há uma exacerbação óbvia desta posição durante o tratamento, com um retorno gradual à posição original, porém parece que esses côndilos mantêm-se anteriorizados mesmo após a remoção do aparelho (grupo III). O tempo após a remoção do aparelho poderia ser um fator decisivo para essas observações. Há uma preocupação óbvia da classe ortodôntica em relação à estabilidade a longo prazo desses tratamentos ortopédicos. Independente do tempo passado após a remoção do aparelho protrusivo, a utilização de aparelho fixo e a obtenção de uma intercuspidação mais efetiva (grupo III apresentou quantidade significante maior de contatos oclusais em $\mathrm{MIH}$ ) parecem ser responsáveis, associados a uma adaptação muscular, pela estabilidade do caso. Os autores ainda constataram grande variabilidade do posicionamento condilar no presente estudo, fato esse em concordância com a literatura em amostras de pacientes assintomáticos ${ }^{1,34}$.

No presente trabalho, em que a concentricidade condilar foi relacionada à prevalência de DTM, não foi possível se estabelecer uma associação entre essas variáveis, como podemos observar na figura 3 . Isso significa que, mesmo diante de diferenças na posição condilar entre os grupos estudados, essa excentricidade condilar não aumentou as chances dos jovens em tratamento com o Bionator apresentarem sinais e sintomas de DTM.

Mesmo que a concentricidade condilar fosse tão importante na etiologia das DTMs, a anteriorização condilar causada pelos aparelhos de avanço mandibular não seria tão danosa às ATMs, uma vez que eventuais discos deslocados poderiam beneficiar-se dessa manobra.

Nesse contexto, quando o deslocamento de disco para anterior for acompanhado de sintomatologia dolorosa, um posicionamento mais para anterior do côndilo poderá ser benéfico no sentido de melhorar os sintomas e a relação côndilo-disco. Com base nesta hipótese, talvez se justifique o fato de muitos trabalhos ${ }^{6,14,30}$ até mesmo indicarem o tratamento com aparelhos ortopédicos para pacientes 
com DTM, porém a estabilidade da recaptura permanente do disco articular ainda carece de comprovação científica.

Baseado no acima exposto, não se pode considerar a protrusão mandibular ortopédica e a conseqüente alteração da posição condilar como fatores de risco para as DTMs. Tais afirmações devem, no entanto, ser interpretadas com cautela, devido ao caráter cíclico e auto-limitante de doenças de etiologia multifatorial como as disfunções temporomandibulares.

\section{CONCLUSÕES}

1. Não houve diferenças na prevalência de sinais e sintomas de DTM entre os pacientes de Classe II antes, durante ou após a terapia com o Bionator;

2. Os fatores oclusais não se relacionaram com os índices de DTM;

3. Os côndilos apresentaram-se significantemente mais anteriorizados no grupo em tratamento, quando comparados ao grupo pré-tratamento;

4. Houve um aumento no espaço articular posterior nos grupos em tratamento ou já tratados;

5. Não houve relação entre concentricidade condilar e sinais e sintomas de DTM.

\title{
Evaluation of condylar position and temporomandibular dysfunction in Class II patients treated with mandibular orthopedic advancement
}

\begin{abstract}
Objective: to evaluate the possible effects of orthopedic mandibular protrusion and condyle position in the prevalence and severity of Temporomandibular Disorders (TMD). Methodology: the sample was composed of 60 individuals with Angle Class II malocclusion divided into three groups depending upon the accomplishment of functional orthopedic treatment (prior, during and after therapy with the Bionator appliance). An anamnestic questionnaire, composed of questions regarding most frequent symptoms was used to classify the sample according to the TMD presence and severity. A clinical examination, including temporomandibular joint (TMJ) and muscle palpation, mandibular range of motion and joint noises analysis was also performed. Serial standard right and left TMJ transcranial images were taken with the teeth in intercuspal position, digitalized and condyle concentricity was determined. Results: one-way ANOVA, Kruskal-Wallis and Chi- square accounted for data analysis. Based on the anamnestic questionnaire, $30 \%$ was considered as having mild TMD, while 3.33\% had moderate TMD, with no difference between groups $(p>0.05)$. Group II (during therapy) has shown significant anterior condyle positioning $(p<0.05)$ when compared to group I, although no association was found between this variable and TMD presence or severity $(p>0.05)$. Conclusion: mandibular orthopedic protrusion was not a risk factor for TMD in the sample studied.
\end{abstract}

Key words: Orthopedics. Temporomandibular dysfunction. Condyle position.

\section{REFERÊNCIAS}

1. ALEXANDER, S. R.; MOORE, R. N.; DuBOIS, L. M. Mandibular condyle position: comparison of articular mountings and magnetic resonance imaging. Am. J. Orthod. Dentofacial Orthop., St. Louis, v. 104, no. 9, p. 230-239, Sept. 1993.

2. AUSTIN, D. G.; PERTES, R. A. Examination of the TMD patient. In: PERTES, R. A.; GROSS, S. G. Clinical management of temporomandibular disorders and orofacial pain. Chicago: Quintessence, 1995. cap. 9, p. 123-160

3. BONILLA-ARAGON, H. et al. Condyle position as a predictor of temporomandibular joint internal derangement. J. Prosthet. Dent. St. Louis, v. 82, no. 2, p. 205-208, 1999.

4. BOSCOLO, F. N.; ALMEIDA, S. M.; PAGANINI, G. A. Estudo da imagem da ATM por duas técnicas radiográficas. Rev. Gaúcha Odontol., Porto Alegre, v. 46, n. 4, p. 222-229, 1998.
5. CARLTON, K. L.; NANDA, R. S. Prospective study of posttreatment changes in the temporomandibular joint. Am. J. Orthod. Dentofacial Orthop., St. Louis, v. 122, no. 5, p. 486-490, Nov. 2002.

6. CLARK, W. J. Twin block functional therapy: applications in dentofacial orthopedics. Turin: Mosby-Wolfe, 1995

7. CONTI, A. C. C. F et al. Relationship between signs and symptoms of temporomandibular disorders and orthodontic treatment: a cross-sectional study. Angle Orthod., Appleton, v. 73, no. 4, p. 411-417, 2003.

8. CONTI, P. C. R. et al. A cross-sectional study of prevalence and etiology of signs and symptoms of temporomandibular disorders in high school and university students. J. Orofac. Pain., Chicago, v. 10, no. 3, p. 254-62, 1996 
9. CONTI, P. C. R. et al. Avaliação da prevalência das dores de cabeça primárias e seu relacionamento com sintomas de desordens temporomandibulares no campus da USP, na cidade de Bauru / SP. Rev. Dental Press Ortodon. Ortop. Facial Maringá, v. 8, n. 2, p. 49-56, mar./abr. 2003.

10. DIBBETS, J. M. H.; VAN DER WEELE, L. T. H. Long-term effect of orthodontic treatment, including extraction, on signs and symptoms attributed to CMD. Eur. J. Orthod., London, v. 14, no. 1, p. 16-20, Feb. 1992

11. FONSÊCA, D. M. Disfunção craniomandibular (DCM): diagnóstico pela anamnese. 1992. 116 f.. Dissertação (Mestrado)Faculdade de Odontologia de Bauru, Universidade de São Paulo, Bauru, 1992

12. GIANELLY, A. A. Orthodontics, condylar position, and TMJ status. Am. J. Orthod. Dentofacial Orthop., St. Louis, v. 95, no. 6, p. 521-523, June 1989.

13. GIANELLY, A. A.; ANDERSON, C. K.; BOFFA, J. Longitudinal evaluation of condylar position in extraction and nonextraction treatment. Am. J. Orthod. Dentofacial Orthop., St. Louis,

v. 100 , no. 5 , p. 416-420, Nov. 1991.

14. GRABER, T. M.; NEWMANN, B. O bionator. In Aparelhos ortodônticos removíveis. 2. ed. São Paulo: Panamericana, 1987. cap. 12, p. 383-402.

15. HELKIMO, M. Studies on function and dysfunction of the masticatory system. I- An epidemiological investigation of symptoms of dysfunction in lapps in north of Finland. Proc. Fin. Dent Soc., Helsinki, v. 70, no. 4, p. 37-49, Apr. 1974.

16. HELKIMO, M. Index for anamnestic and clinical dysfunction and occlusal state. Proc. Fin. Dent Soc., Helsinki, v. 70, no. 4 p. 101-121, Apr. 1974

17. HELKIMO, M. Studies on function and dysfunction of the masticatory system. III: analysis anamnetic and clinical recordings of dysfunction with the aid of index. Swed. Dent. J. Stockholm, v. 67, no. 3, p. 15-82, Mar. 1974.

18. HENRIKSON, T.; NILNER, M. Temporomandibular disorders and need of stomatognathic treatment in orthodontically treated and untreated girls. Eur. J. Orthod., London, v. 22, no. 3, p. 283-292, June 2000.

19. HENRIKSON, T.; NILNER, M.; KUROL, J. Signs of temporomandibular disorders in girls receiving orthodontic treatment: a prospective and longitudinal comparisons with untreated Class II malocclusion and normal occlusion subjects. Eur. J. Orthod., London, v. 22, no. 3, p. 271-281, June 2000.

20. HENRIKSON, T.; NILNER, M.; KUROL, J. Symptoms and signs of temporomandibular disorders before, during and after orthodontic treatment. Swed Dent. J., Stockholm, v. 23, no. 5-6, p. 193-207, 1999

21. ISMAIL, Y. H.; ROKNI, A. Radiographic study of condylar position in centric relation and centric occlusion. J. Prosth. Dent. St. Louis, v. 43, no. 3, p. 328-330, Mar. 1980.

22. JANSON, M.; HASUND, A. Functional problems in orthodontic patients out of retention. Eur. J. Orthod., London, v. 3, p. 173-1739, 1981

23. KUNDINGER, K. K. et al. An evaluation of temporomandibular joints and jaw muscles after orthodontic treatment involving premolar extractions. Am. J. Orthod. Dentofacial Orthop. St. Louis, v. 100 , no. 2, p. 110-115, Aug. 1991.

24. LUECKE, P. E.; JOHNSTON JUNIOR, L. E. The effect of maxillary first premolar extraction and incisor retraction on mandibular position: testing the central dogma of "functional orthodontics". Am. J. Orthod. Dentofacial Orthop., St. Louis, v. 101, no. 1, p. 4-12, Jan. 1992.

25. MAJOR, P. et al. Condyle displacement associated with premolar extraction and nonextraction orthodontic treatment of Class I malocclusion. Am. J. Orthod. Dentofacial Orthop. St. Louis, v. 112, no. 4, p. 435-440, Oct. 1997

26. MCNAMARA JUNIOR, J. A.; SELIGMAN, D. A.; OKESON, J. P. Occlusion, orthodontic treatment, and temporomandibular disorders: a review. J. Orofac. Pain, Chicago, v. 9, no. 1, p. 73-90, 1995.
27. MIKHAIL, M. G.; ROSEN, H. The validity of temporomandibular joint radiographs using the head positioner. J. Prosth. Dent., St. Louis, v. 42, no. 4, p. 441-446, Oct. 1979.

28. MYERS, D. R. et al. Condylar position in children with functional posterior crossbites: before and after crossbite correction. Pediatr. Dent., Chicago, v. 2, no. 3, p. 190-194, 1980.

29. NIELSEN, L.; MELSEN, B.; TERP, S. TMJ function and the effects on the masticatory system on 14-16 year-old Danish children in relation to orthodontic treatment. Eur. J. Orthod. London, v. 12, no. 3, p. 254-262, Aug. 1990.

30. OWEN, A. H. Orthopedic/orthodontic treatment of craniomandibular pain dysfunction. Part B: treatment flow. Sheet, anterior disk displacement, and case histories. J. Craniomandibular Pract., Chatanooga, v. 6, no. 1, p. 48-63, Jan. 1988.

31. PANCHERZ, H. The Herbst appliance - its biologic effect and clinical use. Am. J. Orthod., St. Louis, v. 87, p. 1-20, 1985.

32. PEREIRA, L. J. Avaliação ultra-sonográfica e tomográfica da articulação temporomandibular em adolescentes. 2003. 101 f. Dissertação (Mestrado)-Faculdade de Odontologia de Piracicaba, Universidade Estadual de Campinas, Piracicaba, 2003.

33. PULLINGER, A.; HOLLENDER, L. Variation in condyle-fossa relationships according to different methods of evaluation in tomograms. .J. Oral Surg., Chicago, v. 62, p. 719-727, 1986.

34. PULLINGER, A. G. et al. A tomografic study of mandibular condyle position in an asymptomatic population. J. Prosth. Dent., St. Louis, v. 53, no. 5, p. 706-713, May 1985

35. REN, Y. F.; ISBERG, A.; WESTESSON, P. L. Condyle position in the temporomandibular joint. Comparison between asymptomatic volunteers with normal disk position and patients with disk displacement. J. Oral Surg., Chicago, v. 80, no. 1, p. 101-107, 1995.

36. RUF, S.; PANCHERZ, H. Does bite-jumping damage the TMJ? A prospective longitudinal clinical and MRI study of Herbst patients. Angle Orthod., Appleton, v. 70, no. 3, p. 183-199, June 2000.

37. RUF, S.; WÜSTEN, B.; PANCHERZ, H. Temporomandibular joint effects of activator treatment: a prospective longitudinal magnetic resonance imaging and clinical study. Angle Orthod., Appleton, v. 72, no. 6, p. 527-540, Dec. 2002.

38. SADOWSKY, C.; BeGOLE, E. A. Long-term status of temporomandibular joint function and functional occlusion after orthodontic treatment. Am. J. Orthod., St. Louis, v. 78, no. 2 p. 201-212, Aug. 1980.

39. SADOWSKY, C.; POLSON, A. M. Temporomandibular disorders and functional occlusion after orthodontic treatment: result of two long-term studies. Am. J. Orthod. Dentofacial Orthop., St. Louis, v. 86, no. 5, p. 386-390, Nov. 1984

40. SOLBERG, W. K.; WOO, M. W.; HOUSTON, J. B. Prevalence of mandibular dysfunction in young adults. J. Am. Dent. Assoc. Chicago, v. 98, p. 25-34, Jan. 1979

41. VAN SICKELS, J. E.; BIANCO JR., H. J.; PIFER, R. G. Transcranial radiographs in the evaluation of craniomandibular disorders. J. Prosth. Dent., St. Louis, v. 49, no. 2, p. 244-249, Feb. 1983.

42. WEINBERG, L. A. Role of condylar position in TMJ dysfunction- pain syndrome. J. Prosth. Dent., St. Louis, v. 41, no. 6 , p. 636-643, June 1979.

43. WITZIG, J. W.; SPAHL, T. J. The clinical management of basic maxillofacial orthopedic appliances. Hong Kong: Year Book Medical, 1987

44. WYATT, W.E. Preventing adverse effects on the temporomandibular joint through orthodontic treatment. Am. J. Orthod. Dentofacial Orthop., St. Louis, v. 91, no. 6, p.493-499, June 1987.

\section{Endereço para correspondência}

Ana Cláudia de Castro Ferreira Conti

Rua Renato Tâmbara 2-147 - Residencial Samambaia

CEP: 17.018-100 - Bauru / SP

E-mail: accfconti@uol.com.br 